Commun.Fac.Sci.Univ.Ank.Series A 1

Volume 63, Number 1, Pages 35-41 (2014)

ISSN $1303-5991$

\title{
NEW GENERATING FUNCTIONS FOR THE KONHAUSER MATRIX POLYNOMIALS
}

\author{
ESRA ERKUŞ - DUMAN AND BAYRAM ÇEKİM
}

\begin{abstract}
Varma et. al. [Ars Combin. 100 (2011) 193-204] introduced the concept of the Konhauser matrix polynomials. In this paper, we obtain some generating functions for these matrix polynomials. Finally, we focus on some special cases.
\end{abstract}

\section{INTRODUCTION}

In 2011, Varma et. al. defined the pair of the Konhauser matrix polynomials as follows:

$$
\begin{aligned}
& Z_{n}^{(A, \lambda)}(x ; k)=\frac{\Gamma(A+(k n+1) I)}{n !} \sum_{r=0}^{n}(-1)^{r}\left(\begin{array}{l}
n \\
r
\end{array}\right) \Gamma^{-1}(A+(k r+1) I)(\lambda x)^{k r} \\
& Y_{n}^{(A, \lambda)}(x ; k)=\frac{1}{n !} \sum_{r=0}^{n} \frac{(\lambda x)^{r}}{r !} \sum_{s=0}^{r}(-1)^{s}\left(\begin{array}{l}
r \\
s
\end{array}\right)\left(\frac{1}{k}((s+1) I+A)\right)_{n}
\end{aligned}
$$

where $A$ is a matrix in $\mathbb{C}^{N \times N}$ satisfying the condition

$$
\operatorname{Re}(\mu)>-1 \text { for every } \mu \in \sigma(A),
$$

$\lambda$ is a complex number with $\operatorname{Re}(\lambda)>0$ and $k \in \mathbb{Z}^{+}$(see [5]). Here, for any matrix $A$ in $\mathbb{C}^{N \times N}$, Pochhammer symbol is defined by

$$
(A)_{n}=A(A+I) \ldots(A+(n-1) I), \quad n \geq 1,(A)_{0}=I .
$$

They show that the Konhauser matrix polynomials $Z_{n}^{(A, \lambda)}(x ; k)$ and $Y_{n}^{(A, \lambda)}(x ; k)$ are biorthogonal with respect to the weight matrix function $x^{A} e^{-\lambda x}$. Furthermore

Received by the editors Nov. 18, 2013, Accepted: May 15, 2014.

2000 Mathematics Subject Classification. Primary 33C45 ; Secondary 15A60.

Key words and phrases. Konhauser matrix polynomials, multilinear generating matrix function, multilateral generating matrix function, jacobi matrix polynomials. 
they derive the following generating matrix functions:

$$
\begin{aligned}
& (1-w)^{-\frac{1}{k}(A+I)} \exp \left[-\lambda x\left\{(1-w)^{-\frac{1}{k}}-1\right\}\right] \\
= & \sum_{n=0}^{\infty} Y_{n}^{(A, \lambda)}(x ; k) w^{n} ;|w|<1
\end{aligned}
$$

for the polynomials $Y_{n}^{(A, \lambda)}(x ; k)$ and

$$
\begin{aligned}
& (1-t)^{-B}{ }_{1} F_{k}\left[\begin{array}{ccc}
B & ; \frac{-t(\lambda x)^{k}}{(1-t) k^{k}}
\end{array}\right] \\
= & \sum_{n=0}^{\infty}(B)_{n} Z_{n}^{(A, \lambda)}(x ; k)\left[(A+I)_{k n}\right]^{-1} t^{n} ;|t|<1,\left|\frac{-t(\lambda x)^{k}}{(1-t) k^{k}}\right|<1
\end{aligned}
$$

for the polynomials $Z_{n}^{(A, \lambda)}(x ; k)$, where $A$ and $B$ are matrices in $\mathbb{C}^{N \times N}$ satisfying the conditions

$$
\left.\begin{array}{c}
\operatorname{Re}(\mu)>-1 \text { for every } \mu \in \sigma(A) \\
A B=B A .
\end{array}\right\}
$$

and ${ }_{1} F_{k}$ is defined as

$$
{ }_{1} F_{k}\left[\begin{array}{ccc}
B & & \\
A_{1} & , \ldots, & A_{k}
\end{array}\right]=\sum_{n=0}^{\infty} \frac{(B)_{n}}{n !}\left[\left(A_{k}\right)_{n}\right]^{-1} \ldots\left[\left(A_{1}\right)_{n}\right]^{-1} x^{n}
$$

for $A_{1}, \ldots, A_{k}$ and $B$ are matrices in $\mathbb{C}^{N \times N}$ satisfying condition $A_{i}+s I$ is invertible for $s \in \mathbb{N}, i=1, \ldots, k$, see [5]. In the present paper, we obtain multilinear and multilateral generating functions for the pair of the Konhauser matrix polynomials. Some special cases are also given.

\section{MULTilinear AND MULTilateral GENERATing matrix FUnCtions}

In this section, we give theorems which derive several substantially more general families of bilinear, bilateral generating functions for the Konhauser matrix polynomials defined by (1.1) and (1.2). Using the similar method considered in [1, 2, 3, 4], we obtain the main theorems.

Theorem 2.1. Corresponding to a non-vanishing function $\Omega_{\mu}\left(y_{1}, \ldots, y_{s}\right)$ of complex variables $y_{1}, \ldots, y_{s}(s \in \mathbb{N})$ and of complex order $\mu$, let

$$
\Lambda_{\mu, \nu}\left(y_{1}, \ldots, y_{s} ; z\right):=\sum_{k=0}^{\infty} a_{k} \Omega_{\mu+\nu k}\left(y_{1}, \ldots, y_{s}\right) z^{k} ;\left(a_{k} \neq 0, \mu, \nu \in \mathbb{C}\right)
$$


and

$$
\Theta_{n, p, \mu, \nu}\left(x ; y_{1}, \ldots, y_{s} ; \zeta\right):=\sum_{l=0}^{[n / p]} a_{l} Y_{n-p l}^{(A, \lambda)}(x, k) \Omega_{\mu+\nu l}\left(y_{1}, \ldots, y_{s}\right) \zeta^{l}
$$

where $A$ is a matrix in $\mathbb{C}^{N \times N}$ satisfying the conditions in (1.6), $n, p \in \mathbb{N}$ and (as usual) $[\alpha]$ represents the greatest integer in $\alpha \in \mathbb{R}$. Then, for $|t|<1$ and $\operatorname{Re}(\lambda)>0$, we have

$$
\begin{aligned}
& \sum_{n=0}^{\infty} \Theta_{n, p, \mu, \nu}\left(x ; y_{1}, \ldots, y_{s} ; \frac{\eta}{t^{p}}\right) t^{n} \\
= & (1-t)^{-\frac{1}{k}(A+I)} \exp \left[-\lambda x\left\{(1-t)^{-\frac{1}{k}}-1\right\}\right] \Lambda_{\mu, \nu}\left(y_{1}, \ldots, y_{s} ; \eta\right) .
\end{aligned}
$$

Proof. For convenience, let $S$ denote the first member of the assertion (2.3) of Theorem 2.1. Plugging the polynomials

$$
\Theta_{n, p, \mu, \nu}\left(x ; y_{1}, \ldots, y_{s} ; \frac{\eta}{t^{p}}\right),
$$

which comes from (2.2) into the left-hand side of (2.3), we obtain

$$
S=\sum_{n=0}^{\infty} \sum_{l=0}^{[n / p]} a_{l} Y_{n-p l}^{(A, \lambda)}(x, k) \Omega_{\mu+\nu l}\left(y_{1}, \ldots, y_{s}\right) \eta^{l} t^{n-p l} .
$$

Upon changing the order of summation in (2.4), if we replace $n$ by $n+p l$, we can write

$$
\begin{aligned}
S & =\sum_{n=0}^{\infty} \sum_{l=0}^{\infty} a_{l} Y_{n}^{(A, \lambda)}(x, k) \Omega_{\mu+\nu l}\left(y_{1}, \ldots, y_{s}\right) \eta^{l} t^{n} \\
& =\left(\sum_{n=0}^{\infty} Y_{n}^{(A, \lambda)}(x, k) t^{n}\right)\left(\sum_{l=0}^{\infty} a_{l} \Omega_{\mu+\nu l}\left(y_{1}, \ldots, y_{s}\right) \eta^{l}\right) \\
& =(1-t)^{-\frac{1}{k}(A+I)} \exp \left[-\lambda x\left\{(1-t)^{-\frac{1}{k}}-1\right\}\right] \Lambda_{\mu, \nu}\left(y_{1}, \ldots, y_{s} ; \eta\right),
\end{aligned}
$$

which completes the proof of Theorem 2.1.

Theorem 2.2. Corresponding to a non-vanishing function $\Omega_{\mu}\left(y_{1}, \ldots, y_{s}\right)$ of complex variables $y_{1}, \ldots, y_{s}(s \in \mathbb{N})$ and of complex order $\mu$, let

$$
\Lambda_{\mu, \nu}\left(y_{1}, \ldots, y_{s} ; z\right):=\sum_{k=0}^{\infty} a_{k} \Omega_{\mu+\nu k}\left(y_{1}, \ldots, y_{s}\right) z^{k} ;\left(a_{k} \neq 0, \mu, \nu \in \mathbb{C}\right)
$$


and

$$
\begin{aligned}
& \Theta_{n, p, \mu, \nu}\left(x ; y_{1}, \ldots, y_{s} ; \zeta\right) \\
= & \sum_{l=0}^{[n / p]} a_{l} \Omega_{\mu+\nu l}\left(y_{1}, \ldots, y_{s}\right)(B)_{n-p l} Z_{n-p l}^{(A, \lambda)}(x, k)\left[(A+I)_{k(n-p l)}\right]^{-1} \zeta^{l},
\end{aligned}
$$

where $A$ and $B$ are matrices in $\mathbb{C}^{N \times N}$ such that $A$ satisfies condition in (1.6), $n, p \in$ $\mathbb{N}$, and (as usual) $[\alpha]$ represents the greatest integer in $\alpha \in \mathbb{R}$. Then, for $|t|<1$, $\left|\frac{-t(\lambda x)^{k}}{(1-t) k^{k}}\right|<1$ and $\operatorname{Re}(\lambda)>0$, we have

$$
\begin{aligned}
& \sum_{n=0}^{\infty} \Theta_{n, p, \mu, \nu}\left(x ; y_{1}, \ldots, y_{s} ; \frac{\eta}{t^{p}}\right) t^{n} \\
= & \Lambda_{\mu, \nu}\left(y_{1}, \ldots, y_{s} ; \eta\right)(1-t)^{-B}{ }_{1} F_{k}\left[\begin{array}{ccc}
B & \\
& ; \frac{-t(\lambda x)^{k}}{(1-t) k^{k}}
\end{array}\right] .
\end{aligned}
$$

Proof. The proof is similar to Theorem 2.1.

Now, we obtain some special cases for generating functions.

Firstly, if we set $\Omega_{\mu+\nu k}(y)=P_{\mu+\nu k}^{(C, D)}(y)\left(\mu, \nu \in \mathbb{N}_{0}\right)$ for $s=1$ in Theorem 2.1, where the Jacobi matrix polynomials $P_{n}^{(C, D)}(y)$ are defined by means of the generating function in [7]:

$$
\begin{aligned}
\sum_{n=0}^{\infty} P_{n}^{(C, D)}(x) t^{n}= & F_{4}\left(I+D, I+C ; I+C, I+D ; \frac{(x-1) t}{2}, \frac{(x+1) t}{2}\right)(28) \\
& \left(\sqrt{\frac{(x-1) t}{2}}+\sqrt{\frac{(x+1) t}{2}}<1\right)
\end{aligned}
$$

where $C$ and $D$ are matrices in $\mathbb{C}^{N \times N}$ satisfying the spectral conditions $\operatorname{Re}(z)>-1$ for each eigenvalue $z \in \sigma(C)$, and $\operatorname{Re}(\eta)>-1$ for each eigenvalue $\eta \in \sigma(D)$, $C D=D C$ and $F_{4}(A, B ; C, D ; x, y)$ is defined by

$$
F_{4}(A, B ; C, D ; x, y)=\sum_{n, k=0}^{\infty}(A)_{n+k}(B)_{n+k}(D)_{n}^{-1}(C)_{k}^{-1} \frac{x^{k} y^{n}}{k ! n !},
$$

where $C+n I$ and $D+n I$ are invertible for every integer $n \geq 0$ in $\sqrt{x}+\sqrt{y}<1$. Then we obtain the following example which provides a class of bilateral generating functions for the Jacobi matrix polynomials and the Konhauser matrix polynomials $Y_{n}^{(A, \lambda)}(x, k)$. 
Example 2.3. Taking $a_{l}=1, \mu=0, \nu=1$ and $|t|<1$, we have

$$
\begin{aligned}
& \sum_{n=0}^{\infty} \sum_{l=0}^{[n / p]} Y_{n-p l}^{(A, \lambda)}(x, k) P_{l}^{(C, D)}(y) \eta^{l} t^{n-p l} \\
= & (1-t)^{-\frac{1}{k}(A+I)} \exp \left[-\lambda x\left\{(1-t)^{-\frac{1}{k}}-1\right\}\right] \\
& \times F_{4}\left(I+D, I+C ; I+C, I+D ; \frac{(y-1) \eta}{2}, \frac{(y+1) \eta}{2}\right),
\end{aligned}
$$

where $\sqrt{\frac{(y-1) \eta}{2}}+\sqrt{\frac{(y+1) \eta}{2}}<1$.

If we take $\Omega_{\mu+\nu k}(y)=C_{\mu+\nu k}^{D}(y)\left(\mu, \nu \in \mathbb{N}_{0}\right)$ for $s=1$ in Theorem 2.2, where the Gegenbauer matrix polynomials $C_{n}^{D}(y)$ are defined by means of the generating function in [6]:

$$
\sum_{n=0}^{\infty}\left[(2 D)_{n}\right]^{-1} C_{n}^{D}(x) t^{n}=\exp (x t){ }_{0} F_{1}\left(-; D+\frac{I}{2} ; \frac{1}{4} t^{2}\left(x^{2}-1\right)\right),
$$

where $D$ is a matrix in $\mathbb{C}^{N \times N}$ satisfying the spectral condition $\left(\frac{-z}{2}\right) \notin \sigma(D)$ for each eigenvalue $z \in \mathbb{Z}^{+} \cup\{0\}$, then we obtain the following example which provides a class of bilateral generating functions for the Gegenbauer matrix polynomials $C_{n}^{D}(x)$ and the Konhauser matrix polynomials $Z_{n}^{(A, \lambda)}(x, k)$.

Example 2.4. Taking $a_{l}=\left[(2 D)_{l}\right]^{-1}, \mu=0, \nu=1$, we have

$$
\begin{aligned}
& \sum_{n=0}^{\infty} \sum_{l=0}^{[n / p]}\left[(2 D)_{l}\right]^{-1} C_{l}^{D}(y)(B)_{n-p l} Z_{n-p l}^{(A, \lambda)}(x, k)\left[(A+I)_{k(n-p l)}\right]^{-1} \eta^{l} t^{n-p l} \\
= & \exp (y \eta)_{0} F_{1}\left(-; D+\frac{I}{2} ; \frac{1}{4} \eta^{2}\left(y^{2}-1\right)\right) \\
& \times(1-t)^{-B}{ }_{1} F_{k}\left[\begin{array}{cc}
B & ; \frac{-t(\lambda x)^{k}}{(1-t) k^{k}}
\end{array}\right],
\end{aligned}
$$

where $A$ and $B$ are matrices in $\mathbb{C}^{N \times N}$ such that $A$ satisfies conditions in (1.6).

Substituting $\Omega_{\mu+\nu k}(y)=Y_{\mu+\nu k}^{\left(C, \lambda_{2}\right)}\left(y, k_{2}\right)\left(\mu, \nu \in \mathbb{N}_{0}\right)$ for $s=1$ in Theorem 2.2, then we can give the following example which provides a class of bilateral generating functions for the pair of the Konhauser matrix polynomials. 
Example 2.5. Taking $a_{l}=1, \mu=0, \nu=1$ and $|t|<1$, we have

$$
\begin{aligned}
& \sum_{n=0}^{\infty} \sum_{l=0}^{[n / p]} Y_{l}^{\left(C, \lambda_{2}\right)}\left(y, k_{2}\right)(B)_{n-p l} Z_{n-p l}^{(A, \lambda)}(x, k)\left[(A+I)_{k(n-p l)}\right]^{-1} \eta^{l} t^{n-p l} \\
& =(1-\eta)^{-\frac{1}{k_{2}}(C+I)} \exp \left[-\lambda_{2} y\left\{(1-\eta)^{-\frac{1}{k_{2}}}-1\right\}\right] \\
& \times(1-t)^{-B}{ }_{1} F_{k}\left[\begin{array}{cccc}
B & & ; \frac{-t(\lambda x)^{k}}{(1-t) k^{k}} \\
\frac{1}{k}(A+I) & , \ldots, & \frac{1}{k}(A+k I)
\end{array}\right],
\end{aligned}
$$

where $C$ is a matrix in $\mathbb{C}^{N \times N}$ satisfying the condition

$$
\operatorname{Re}(\mu)>-1 \text { for every } \mu \in \sigma(C),
$$

$\lambda_{2}$ is a complex number with $\operatorname{Re}\left(\lambda_{2}\right)>0, k_{2} \in \mathbb{Z}^{+}$and $|\eta|<1$.

Also setting $\Omega_{\mu+\nu l}(y)=Y_{\mu+\nu l}^{\left(C, \lambda_{2}\right)}\left(y, k_{2}\right)\left(\mu, \nu \in \mathbb{N}_{0}\right)$ for $s=1$ in Theorem 2.1, we have the following example which provides a class of bilinear generating functions for the Konhauser matrix polynomials $Y_{n}^{(A, \lambda)}(x, k)$.

Example 2.6. Taking $a_{l}=1, \mu=0, \nu=1$ and $|t|<1$, we have

$$
\begin{aligned}
& \sum_{n=0}^{\infty} \sum_{l=0}^{[n / p]} Y_{n-p l}^{(A, \lambda)}(x, k) Y_{l}^{\left(C, \lambda_{2}\right)}\left(y, k_{2}\right) \eta^{l} t^{n-p l} \\
= & (1-t)^{-\frac{1}{k}(A+I)} \exp \left[-\lambda x\left\{(1-t)^{-\frac{1}{k}}-1\right\}\right] \\
& \times(1-\eta)^{-\frac{1}{k_{2}}(C+I)} \exp \left[-\lambda_{2} y\left\{(1-\eta)^{-\frac{1}{k_{2}}}-1\right\}\right]
\end{aligned}
$$

where $C$ is a matrix in $\mathbb{C}^{N \times N}$ satisfying the condition

$$
\operatorname{Re}(\mu)>-1 \text { for every } \mu \in \sigma(C),
$$

$\lambda_{2}$ is a complex number with $\operatorname{Re}\left(\lambda_{2}\right)>0, k_{2} \in \mathbb{Z}^{+}$and $|\eta|<1$.

Similarly, in Theorem 2.2, if we take $\Omega_{\mu+\nu l}(y)=Z_{\mu+\nu l}^{\left(C, \lambda_{2}\right)}\left(y, k_{2}\right)\left(\mu, \nu \in \mathbb{N}_{0}\right)$, where $C$ is a matrix in $\mathbb{C}^{N \times N}$ satisfying the condition

$$
\operatorname{Re}(\mu)>-1 \text { for every } \mu \in \sigma(C),
$$

$\lambda_{2}$ is a complex number with $\operatorname{Re}\left(\lambda_{2}\right)>0$ and $k_{2} \in \mathbb{Z}^{+}$, then we obtain bilinear generating functions for the Konhauser matrix polynomials $Z_{n}^{(A, \lambda)}(x ; k)$.

Notice that, when the multivariable function $\Omega_{\mu+\nu k}\left(y_{1}, \ldots, y_{s}\right),\left(k \in \mathbb{N}_{0}, s \in \mathbb{N}\right)$, is expressed in terms of several simpler functions of one or more variables, then each suitable choice of the coefficients $a_{k}\left(k \in \mathbb{N}_{0}\right)$ in Theorems 2.1 and 2.2 can be 
shown to yield various classes of multilateral and multilinear generating functions for the Konhauser matrix polynomials defined by (1.1) and (1.2).

\section{REFERENCES}

[1] A. Altın and E. Erkuş, On a multivariable extension of the Lagrange-Hermite polynomials, Integral Transform. Spec. Funct., 17 (2006), 239-244.

[2] E. Erkuş-Duman, Matrix extensions of polynomials in several variables, Util. Math., 85 (2011), 161-180.

[3] B. Çekim and E. Erkuş-Duman, On the g-Jacobi Matrix Functions, Advances in Applied Mathematics and Approximation Theory, Springer Proceedings in Mathematics \& Statistics, 73-84, 2013.

[4] R. Aktaş, B. Çekim and R. Şahin, The matrix version for the multivariable Humbert polynomials, Miskolc Math. Notes, 13 (2) (2012), 197-208.

[5] S. Varma, B. Çekim, F. Taşdelen Yeşildal, On Konhauser matrix polynomials, Ars Combin., 100 (2011), 193-204

[6] K.A.M. Sayyed, M.S. Metwally and R.S. Batahan, Gegenbauer matrix polynomials and second order matrix differential equations, Divulg.Mat., 12 (2) (2004), 101-115.

[7] A. Altın, B. Çekim and E. Erkuş-Duman, Families of generating functions for the Jacobi and related matrix polynomials, Ars Combin. (in press).

Current address: Gazi University, Faculty of Science, Department of Mathematics, Teknikokullar TR-06500, Ankara, TURKEY

E-mail address: eduman@gazi.edu.tr, bayramcekim@gazi.edu.tr

$U R L:$ http://communications.science. ankara.edu.tr/index.php?series=A1 